\title{
Correlation between Neonatal Sepsis and Red Blood Cell Distribution Width (RDW)
}

\author{
Ahmed Mahmoud Abdelmoktader ${ }^{(1)}$,Rehab GalalAbd El-Hamid ${ }^{(1)}$, Mohammed \\ Mansour Abbas ${ }^{(1)}$, Mohammed Sherif Ahmed Salim ${ }^{(1)}$
}

(1) Pediatric Department, Clinical Pathology Department, Faculty of Medicine, Fayoum University, Egypt

\section{Corresponding author: Dr. Mohammed Sherif Ahmed Salim \\ E-mail address: mohsherif272@gmail.com}

Tel: 01066284567

\section{ABSTRACT}

Background: Neonatal sepsis remains a challenge for neonatal care providers.

\begin{abstract}
Aim: This work was aiming to measure red cell distribution width percent (RDW \%) as a marker for neonatal sepsis and to correlate it with other indicators and with a simple clinical scoring system for neonatal sepsis (Griffin score).
\end{abstract}

Methods: This case control study was carried out at neonatal intensive care unit at Fayoum University Hospital and Abshawai central hospital . Ethical Research Committee Approval and written consents were obtained from parents of the neonates 30 neonatal sepsis cases and 30 normal controls. Neonates were subjected to: History taking, clinical examination for manifestations of sepsis. Complete blood count, differential leucocytic count, C- reactive protein, Blood culture, RBS, and determination of RDW \% were done to all neonates.
Results: Our study showed that I/T ratio and RBS was significantly higher in cases than controls ( $\mathrm{p}<0.001)$, but WBCs show no statistically significant difference among cases and controls. Moreover, blood culture was positive in $63.3 \%$ of cases only. Also, CRP was high in $76.7 \%$ of cases and $30 \%$ of controls $(\mathrm{P}=0.01)$, and that the mean RDW \% was higher among cases than controls $(16.4 \pm$ 3.8 and $13.7 \pm 1.6$ respectively) $(\mathrm{P}=0.001)$, with further increase in preterm neonates . Also, there was a significant positive correlation between RDW level Griffin score $(\mathrm{p}=0.001)$. However, there was insignificant difference between RDW and WBCs or I/T ratio but high RDW level associated with raised RBS level of study group.

Conclusion: RDW can serve as a marker and prognostic indicator in neonatal sepsis especially when used together with Griffin score.

\section{KEY WORDS: Neonatal sepsis, RDW, Griffin score.}

\section{INTRODUCTION}

Neonatal sepsis is a major cause of mortality in the developing countries. As the signs and symptoms of sepsis are non-specific, early diagnosis poses a challenge to the clinicians .Blood culture has been commonly used as a traditional approach for the detection of sepsis. However, blood cultures are time- consuming and at least $2-5$ days of culture is necessary to identify the organism in the blood. Moreover, the sensitivity of blood cultures declines significantly if antibiotic therapy has been initiated, or when fastidious or slow growing pathogens are cultured (1). 
Therefore, other test, such as a complete blood count $(\mathrm{CBC})$ is done along with the blood culture to determine sepsis.

Conventional screening tests such as total and differential leukocyte counts, band cells, neutrophil counts and rapid immunological tests like C-reactive protein (CRP) assays may help in the diagnosis of septicemias; however, they lack the capacity to predict the severity of sepsis (2).

\section{Red Cell Distribution Width (RDW)} indicates heterogeneity of erythrocyte volume in circulation and routinely it is reported as a component of $\mathrm{CBC}$ without incurring additional cost. RDW is calculated by dividing standard deviation of red blood cell (RBC) volume by mean corpuscular volume (MCV) and multiplying the product by 100 . Higher RDW values indicate variations of RBC volume. It is mainly used for the differential diagnosis of microcytic anemia (3).

\section{PATIENTS AND METHODS}

Study design: This study was a case control study.

Study population and sampling; This study was conducted in the neonatal intensive care unit (NICU) in Fayoum University Hospital and Abshawai central hospital. Sixty neonates were enrolled in this study, thirty neonates with suspected neonatal sepsis according to clinical and laboratory data, and for controls thirty healthy neonates with age and sex matched with the case group.

Inclusion criteria: 1-Neonates who were $>28$ weeks of gestational age.

\section{Exclusion criteria:}

1- Neonates who had lethal congenital anomalies and severe encephalopathy

2-Neonates who were large for gestational age (birth weight_90 $9{ }^{\text {th }}$ percentile).

3- Maternal medications that may affect the fetal hemopoietic system.

4- Significant antenatal bleeding in mother.
RDW has been extensively studied in the differential diagnosis of anemia and in patients with congestive heart failure, acute myocardial infarction, pulmonary embolism, and pneumonia (4) .

Recent studies also suggest that RDW is a useful biomarker of disease severity in critically ill patients and an increased RDW is an independent predictor of mortality in sepsis (5).

In Egypt, neonatal sepsis is considered a big problem as with lack of infection control measures and inadequate nursing staff, the incidence range increases more than the documented incidence. Therefore, the present study was undertaken to look for the association of RDW with neonatal sepsis and its role in predicting mortality (6).

5- Positive family history in terms of hematologic diseases such as thalassemias.

6-Neonates with any medical conditions such as hydrops fetalis, intrauterine transfusions, grade 3 or 4 intraventricular hemorrhage, fetomaternal hemorrhage, cyanotic congenital heart disease, shock were excluded as they Can independently influence RDW other than sepsis.

7- Neonates born to mothers with severe anaemia (haemoglobin $<8 \mathrm{mg} / \mathrm{dl}$ ).

8- Neonates with low Apgar score $(\leq 7)$ within 5 minutes after delivery.

9- Neonates born to mothers with multiple pregnancies.

\section{All cases were subjected to the following:}

- Full maternal history taking including maternal age, gravity\& parity ,and medical \& obstetric history ,details of labor with stress on any prenatal hazards e.g. pre-eclampsia ,PROM , antepartum hemorrhage ,fever ,UTI ,chorioamnionitis. 
- Detailed perinatal history of the neonate including gestational age, mode of delivery .early postnatal cyanosis or using any invasive procedures.

- Clinical assessment as regard full general and systemic assessment including :

-Vital signs: heart rate, respiratory rate, Temperature and blood pressure.

-Anthropometric measures: weight, length and head circumference.

- Neonatal reflexes e.g. Moro and Suckling reflexes and
-Organ system examination particularly for signs of sepsis e.g. lethargy, hypotonia, poor perfusion, poor reflexes, mottling of skin, hypothermia and intolerance to feed

- Laboratory assessment in the form of: CBC (including TLC, RDW and I/T ratio), CRP, Blood culture and Random blood sugar.

We used a clinical scoring system (Griffin et al clinical score for symptoms and signs of sepsis) (7) for selection of cases which includes:

\begin{tabular}{|l|l|}
\hline Signs & points \\
\hline $\begin{array}{l}\text { Feeding intolerance (feedings held for greater than } 24 \text { hours) in an infant } \\
\text { who had been tolerating advancing or full feeds for three days. }\end{array}$ & 2 \\
\hline Severe apnea requiring positive pressure ventilation & 2 \\
\hline $\begin{array}{l}50 \% \text { increase in number of apneic episodes over a 24 hour period in an } \\
\text { infant who had been extubated and stable for three days }\end{array}$ & 2 \\
\hline Immature/total neutrophil (I:T) ratio greater than 0.2 & 2 \\
\hline Increase in ventilatory support and FiO2 by 25\% from baseline & 1 \\
\hline Lethargy or hypotonia & 1 \\
\hline Temperature instability & 1 \\
\hline Hyperglycemia (>180 mg/dL) & 1 \\
\hline Abnormal white blood cell count (>25,000 or<5,000) & 1 \\
\hline
\end{tabular}

Total score $=$ SUM (points for all 7 parameters)..Interpretation $: \bullet$ minimum score: $0 \bullet$ Maximum score: $13 \cdot$ A score $\geq 2$ was associated with sepsis

CBC was performed on automated cell counter (coulter) with the differential count done on Leishmania- Giemsa stained blood film. Estimation of CRP level in the done using latex agglutination test. Blood Cultures were performed using
BACTEC peds blood culture bottles and the BACTEC-9050 instrument.

For collection of blood culture, the resident doctor wear sterile gloves prior to the procedure and prepare a patch of skin 
approximately $5-\mathrm{cm}$ in diameter over the proposed veni-puncture site. This area is cleansed thoroughly with alcohol, followed by povidone iodine, and followed again by alcohol. povidone iodine applied in concentric circles moving outward from the Centre. the skin is allowed to dry for at least 1 minute before the

\section{RESULTS}

In the current study, we studied 60neonates, 30 with clinical sepsis and 30 were non-sepsis neonates. As regarding sex, $70 \%$ of cases were males and $30 \%$ were females. So, sepsis was more prevalent among males, But the difference was not statistically significant $(\mathrm{P}$ $=0.2$ ). $60 \%$ of cases were full term and $40 \%$ were preterm with increased incidence of sepsis among preterm cases $(p=0.005)$ versus controls. Also, sepsis was more prevalent among low birth weight (LBW) neonates (main weight for controls $=2.9$ but for cases $=$ 2.4 with $\mathrm{p}=0.002)$. Also the percentage of sepsis was high in neonates born by cesarean section than by vaginal delivery with no statistically significant difference $(\mathrm{p}=0.6)$. There was statistically significant difference with p-value $<0.05$ between study groups as regards RBS, I/T, and RDW level with high mean among cases (table 1 ).

There was statistically significant difference with $\mathrm{p}$-value $<0.05$ between study groups as regards Griffin score level with high sample is collected .one $\mathrm{mL}$ sample of blood is adequate for blood culture bottle containing 5-10 $\mathrm{mL}$ of culture media since samples collected from indwelling lines and catheters are likely to be contaminated , cultures only collected from a fresh vein-puncture site. mean among cases. Regarding other markers for diagnosing of sepsis, CRP level was elevated only in $30 \%$ of controls, and $76.7 \%$ of cases with a statistically significant difference ( $\mathrm{P}=0.01)$ (table 3 ). Also, 63.3\% of cases showed positive blood culture while no of controls showed positive blood culture with statistically significant difference $(\mathrm{P}<0.001)$ (table 4).

On the other hand, we observed high level of RDW among positive culture ( $p$-value $<0.05)$. On the other hand there was statistically significant positive correlation with p-value <0.05 between RDW level and each of RBS and level Griffin score, which indicated increase in RBS and level Griffin score will be associated with increase in RDW level among whole study group (table 5). Moreover we observed that there was statistically significant positive correlation with $p$-value $<0.05$ between Griffin score and each of RBS, and I/T( table 6) .

Table (1): Comparisons of RBS, I/T, and RDW in different study groups:

\begin{tabular}{|l|c|c|c|c|c|c|}
\hline \multirow{2}{*}{ Variables } & \multicolumn{2}{|c|}{$\begin{array}{c}\text { Control } \\
(\mathrm{n}=30)\end{array}$} & \multicolumn{2}{c|}{$\begin{array}{c}\text { Cases } \\
(\mathrm{n}=30)\end{array}$} & \multirow{2}{*}{ p-value } & \multirow{2}{*}{ Sig. } \\
\cline { 2 - 6 } & Mean & SD & Mean & SD & & \\
\hline RBS & 89.6 & 31.8 & $\mathbf{1 3 9 . 4}$ & 65.5 & $<\mathbf{0 . 0 0 1}$ & HS \\
\hline TLC & 13.3 & 4.7 & 14.2 & 8.8 & 0.6 & NS \\
\hline I/T & 0.07 & 0.04 & $\mathbf{0 . 1 7}$ & 0.09 & $<\mathbf{0 . 0 0 1}$ & HS \\
\hline RDW & 13.7 & 1.6 & $\mathbf{1 6 . 4}$ & 3.8 & $\mathbf{0 . 0 0 1}$ & HS \\
\hline
\end{tabular}


Table (2): Comparisons of Griffin score in different study groups: .

\begin{tabular}{|l|c|c|c|c|}
\hline \multirow{2}{*}{ Variables } & \multicolumn{2}{|c|}{ Griffin score } & \multirow{2}{*}{ p-value } & \multirow{2}{*}{ Sig. } \\
\cline { 2 - 3 } & Mean & SD & & \\
\hline Controls & 0.4 & 0.4 & \multirow{2}{*}{$<0.001$} & \multirow{2}{*}{ HS } \\
\hline Cases & $\mathbf{3 . 9}$ & 1.7 & & \\
\hline
\end{tabular}

Table (3): Comparisons of CRP and culture results in different study groups:

\begin{tabular}{|c|c|c|c|c|c|c|}
\hline \multirow[t]{2}{*}{ Variables } & \multicolumn{2}{|c|}{$\begin{array}{c}\text { Control } \\
(\mathrm{n}=30)\end{array}$} & \multicolumn{2}{|c|}{$\begin{array}{l}\text { Cases } \\
(\mathrm{n}=30)\end{array}$} & \multirow[t]{2}{*}{ p-value } & \multirow[t]{2}{*}{ Sig. } \\
\hline & No. & $\%$ & No. & $\%$ & & \\
\hline \multicolumn{7}{|l|}{ CRP } \\
\hline CRP 0 & 21 & $70 \%$ & 7 & $23.3 \%$ & \multirow{6}{*}{0.01} & \multirow{6}{*}{$\mathbf{S}$} \\
\hline CRP 6 & 2 & $6.7 \%$ & 2 & $6.7 \%$ & & \\
\hline CRP 12 & 3 & $10 \%$ & 5 & $16.7 \%$ & & \\
\hline CRP 24 & 2 & $6.7 \%$ & 6 & $20 \%$ & & \\
\hline CRP 48 & 1 & $3.3 \%$ & 7 & $23.3 \%$ & & \\
\hline CRP 96 & 1 & $3.3 \%$ & 3 & $10 \%$ & & \\
\hline \multicolumn{7}{|c|}{ Culture results } \\
\hline Positive & 0 & $0 \%$ & 19 & $63.3 \%$ & \multirow{2}{*}{$<0.001$} & \multirow{2}{*}{ HS } \\
\hline Negative & 30 & $100 \%$ & 11 & $36.7 \%$ & & \\
\hline
\end{tabular}

Table (4): Comparisons of RDW level in different CRP and culture results among cases:

\begin{tabular}{|c|c|c|c|c|}
\hline \multirow{2}{*}{ Variables } & \multicolumn{2}{|c|}{ RDW } & \multirow{2}{*}{ p-value } & \multirow{2}{*}{ Sig. } \\
\hline & Mean & SD & & \\
\hline \multicolumn{5}{|l|}{ CRP } \\
\hline CRP 0 & 15 & 4.3 & \multirow{6}{*}{0.9} & \multirow{6}{*}{ NS } \\
\hline CRP 6 & 14.5 & 1 & & \\
\hline CRP 12 & 15.3 & 2.2 & & \\
\hline CRP 24 & 15.4 & 1.4 & & \\
\hline CRP 48 & 15 & 2.3 & & \\
\hline CRP 96 & 14.5 & 1.7 & & \\
\hline \multicolumn{5}{|l|}{ Culture results } \\
\hline Positive & 16.6 & 4.2 & \multirow{2}{*}{0.008} & \multirow{2}{*}{ HS } \\
\hline Negative & 14.3 & 2.4 & & \\
\hline
\end{tabular}

Table (5): Correlation between RDW and study variables among study group:

\begin{tabular}{|l|c|c|c|}
\hline \multirow{2}{*}{ Variables } & \multicolumn{3}{c|}{ RDW } \\
\cline { 2 - 4 } & R & p-value & Sig. \\
\hline Demographic characters & -0.03 & 0.9 & NS \\
\hline Age (days) & -0.23 & 0.08 & NS \\
\hline weight (kg) & & \\
\hline Blood picture & & \\
\hline
\end{tabular}




\begin{tabular}{|l|c|c|c|}
\hline RBS & $\mathbf{0 . 2 5}$ & $\mathbf{0 . 0 5}$ & S \\
\hline TLC & -0.07 & 0.6 & NS \\
\hline I/T & 0.17 & 0.2 & NS \\
\hline Griffin score & $\mathbf{0 . 4 3}$ & $\mathbf{0 . 0 0 1}$ & HS \\
\hline Griffin Score &
\end{tabular}

Table (6): Correlation between Griffin Score and study variables among cases:

\begin{tabular}{|l|c|c|c|}
\hline \multirow{2}{*}{ Variables } & \multicolumn{3}{c|}{ Griffin score } \\
\cline { 2 - 4 } & r & p-value & Sig. \\
\hline Demographic characters & 0.09 & 0.6 & NS \\
\hline Age (days) & -0.08 & 0.7 & NS \\
\hline weight (kg) & \multicolumn{3}{|l|}{} \\
\hline Blood picture & 0.43 & $\mathbf{0 . 0 1}$ & S \\
\hline RBS & 0.21 & 0.3 & NS \\
\hline TLC & 0.57 & $\mathbf{0 . 0 0 1}$ & HS \\
\hline I/T & &
\end{tabular}

\section{DISCUSSION}

Early identification and timely treatment of sepsis in children is particularly critical in reducing the mortality. New studies found that RDW increase can be used as an important and independent predictive factor of the incidence of deaths caused by various diseases. Meanwhile, RDW values can reflect the degree of overall inflammation and oxidative stress $(8)$.

The authors reported that the combination of baseline RDW value and percent increases in RDW values might be a promising independent prognostic factor in patients with severe sepsis or septic shock (3). We used a more recent and practical clinical scoring system to select our cases, called the Point score for imminent neonatal sepsis (Griffin score), which is developed by (7).
Our results revealed increased incidence of sepsis among preterm cases, these finding were similar to Medhat et al., 2017 (6).

Moreover, the finding of increased $\mathrm{I} / \mathrm{T}$ ratio $(\geq 0.2)$ among cases was similar to Dalia A. Saied., 2017 (9) who reported that neutrophil left shift (I/T ratio $\geq 0.2)$ showed a significant correlation with early clinical signs of sepsis $(\mathrm{P}<.001)$, and higher incidence of LOS (P = .001), Also Lazarus Monica., 2018 (10) revealed that $\mathrm{I} / \mathrm{T}$ ratio was higher in culture positive cases.

Our study illustrated that the mean RDW was significantly higher in cases compared to controls $(\mathrm{P}=0.001)$ (table 1$)$. This finding was in agreement with Jianping et al., 2015 (11) and Medhat et al., 2017 (6) who reported that RDW value of sepsis group $(19.61 \pm 1.48$ 
$\& 18.35 \pm 1.79$ respectively ) was much more higher than that of normal control group. Also Snehal L. Martin et al., 2018 (1) observed that RDW values were higher in the neonatal sepsis cases than those of the control group ( $\mathrm{p}<.001)$. Similarly, Cosar et al., 2017 (3) found in their study that RDW indices were higher than those of the control group $(\mathrm{p}<0.001)$ in term and near-term newborns with EONS. But Abbasoglu et al., 2014 (12) did not find any change in RDW values in neonatal sepsis. However, these studies were retrospective in nature with a small sample size.

We observed high mean Griffin score mean among cases $(\mathrm{p}<0.001)$. This was similar to Griffin et al., 2007 (7) who found highly significant correlations of the clinical score and individual clinical signs of sepsis.

Regarding other markers of diagnosing sepsis, CRP level was elevated only in $30 \%$ of controls, and $76.7 \%$ of cases with a statistically significant difference $(\mathrm{P}=0.01)$ (table 3 ).This finding was in agreement with Sidra et al., 2014 (13), who found that the mean CRP level was significantly higher in patients with sepsis than controls. Also Buch et al., 2011 (14), reported that CRP has high sensitivity and specificity for establishing the diagnosis of neonatal sepsis which is comparable to that of blood culture results. Serial CRP estimation may also of great value in monitoring the degree of response to treatment in clinically infected neonates and thus may guidethe clinicians to estimate duration of antibiotic therapy correctly (Buch et al., 2011) (14).

Blood culture remains the "gold standard" for diagnosing of neonatal sepsis , $63.3 \%$ of cases showed positive blood culture while no of controls showed positive blood culture (table $3)$. Medhat et al.,2017(6) reported that $88 \%$ of cases showed positive blood culture while only $6.7 \%$ of controls showed positive blood culture with statistically significant difference $(\mathrm{P}=0.001)$. This was in agreement with results of Neal et al., 2011 (15), who reported that as many as $60 \%$ of blood cultures would be falsely negative for common neonatal pathogens and this may be explained by the fact that maternal antibiotics given in the majority of preterm deliveries may suppress the growth of bacteria in culture and subsequently give negative blood culture results.

There was no statistically significant difference in RDW level between CRP groups $(\mathrm{p}=0.9)$ among cases (table 4). However, Aksoy et al., 2013 (16) observed a negative correlation between RDW indices and CRP levels of the patients. On the other hand ,Cosar et al., 2017(3) and Ozdogan et al., 2015 (17) reported that there was a significant positive correlation between RDW and serum CRP levels, suggesting that inflammation leads to increased RDW.

However, we observed that the level of RDW was higher among cases with positive blood culture $(\mathrm{p}=0.008)$ (table 4$)$. Our work showed that high level of RDW was associated with increased Griffin score and RBS among the whole study group. (table 5)

Our study revealed that there was no association between Griffin score and age or weight of neonate among cases, But there was a positive correlation between Griffin score and RBS among cases with a significant difference $(\mathrm{p}=0.01)$ (table 6$)$. Also there was a positive correlation between Griffin score and $\mathrm{I} / \mathrm{T}$ ratio among cases with a highly significant difference $(p=0.001)$. On the other hand, there was no statistically significant correlation $(\mathrm{p}=0.3$ ) between Griffin score and TLC among cases.

The main limitations of our study were relatively scarce number of neonates included in the study. Secondly, we did not analyze the levels of IL- 6 and TNF- $\alpha$ and other markers of inflammation which involved in the diagnosis and pathogenesis of sepsis and their correlations with RDW. Finally, the changes of RDW in response to treatments applied were not assessed.

\section{Conclusion}

This study revealed that RDW may become a new indicator for diagnosis and risk stratification of sepsis in newborns due to being simple, less expensive, available and easily repeated. Also, it does not need special laboratory facilities as it is routinely done with CBC especially when used together with 
simple clinical scoring system for imminent clinical neonatal illness. So it may reflect a favorable impact on clinical care. Thus, RDW

\section{REFERENCES}

[1] Snehal L. Martin, SaumilDesaib, RuchiNanavatib, RoshanB.Colaha, KanjakshaGhoshaandMalay B. Mukherjeea.:Red cell distribution width and its association with mortality in neonatal sepsis. The Journal Of Maternal -Fetal \& Neonatal Medicine 2018.

[2] Jajoo M, Kapoor K, Garg LK., et al.:To study the incidence and risk factors of early onset neonatal sepsis in an out born neonatal intensive care unit of India. J ClinNeonatal. 2015;4:91-95.

[3] Cosar H1, Yilmaz O2, Temur M2, OzunOzbay P3, Bulut Y4 and Karakulak M5.: Relationship between Early-Onset Neonatal Sepsis and Red Blood Cell Distribution Width (RDW). J HematolThrombo Dis 2017, 5:2

[4] Jo YH, Kim K, Lee JH., et al.: Red cell distribution widthis a prognostic factor in severe sepsis and septic shock. Am J Emerg Med. 2013; 31:545-548.

[5] Ramby AL, Goodman DM, Wald EL., et al.: Red blood cell distribution width as a pragmatic marker for outcome in pediatric critical illness. PLoS One. 2015;10:e0129258.

[6] Medhat A Saleh, Yasser T Kasem, Hesham H Amin.: Evaluation of neonatal sepsis and assessment of its severity by Red Cell Distribution Width indicator. The Egyptian Journal of Community Medicine Vol. 35 No. 3 July 2017.

[7] Griffin MP, Lake DE, O'Shea TM, Moorman JR.: Heart rate characteristics and clinical signs in neonatal sepsis. Pediatr Res. 2007; 61(2):222-7.

[8] Hunziker S, Stevens J, Howell MD.: Red cell distribution width and mortality in newly hospitalized patients. Am J Med 2012; 125: 283-291.

[9] Dalia A. Saied.,2017.:Can we rely on the neutrophil left shift for the diagnosis of neonatal sepsis? Need for re-evaluation. The may be used as an ideal predictive value for evaluation of sepsis risk, and to evaluate the prognosis of neonatal sepsis.

Gazette of the Egyptian Paediatric Association.DOI: 10.1016/j.epag.2017.12.002.

[10] Lazarus Monica, J. Seth Riti and B. KinnareAmit.:Role of Sepsis Screen Parameters in Early Diagnosis of Neonatal Septicemia.Int.J.Curr.Microbiol.App.Sci (2018) 7(1): 2410-2419

[11] Jianping Chen, Ling Jin, Tong Yang. Clinical study of RDW and prognosis in sepsis newborns.Biomedical Research 2015; 25 (4): 576-579.

[12] Abbasoglu A, Tugcu U, AnukInce D., et al.: PO-0514 Assessment of red cell distribution width in neonatal sepsis as a prognostic factor. Arch Dis Child. 2014; 99:A417.

[13] Sidra Younis, Muhammad Ali Sheikh, Amjad Ali Raza.: 2014 .Diagnostic Accuracy of C - reactive protein in Neonatal Sepsis. J. Bio resource Manage. 1(1): 33-42.

[14] Bunch, A.C., V. Srivastava, H. Kumarand P.S. Jadhav (2011).: Evaluation of haematological profiles in early diagnosis of clinically suspected cases of neonatal sepsis. International Journal of Basic and Applied Medical Sciences, 1: 1-6.

[15] Neal P. R., KleimanM. B., ReynoldsJ. K., AllenS. D., LemonsJ. A., YuP. L.:(2011): Volume of blood submitted for culture from neonates. J. Clin. Microbiol. 24:353-356.

[16] Aksoy HT, Eras Z, Canpolat E, Dilmen U.: (2013).Does red cell distribution width predict mortality in newborns with early sepsis? Am JEmerg Med 31: 1150.

[17] Ozdogan HK, Karateke F, Ozyazıcı S., et al.:The predictive value of red cell distribution width levels on mortality in intensive care patients with community acquired intra-abdominal sepsis. UlusTravmaAcilCerrahiDerg. 2015; 21:352357. 